\title{
Change in timed walk as primary outcome measure of treatment response in HAMLET-P: HAM/TSP MuLticentre Efficacy trial-Prednisolone
}

\author{
Fabiola Martin ${ }^{1 *}$, Eisuke Inoue ${ }^{2}$, Maria Fernanda Rios Grassi ${ }^{3,4}$, Ramon de Almeida Kruschewsky ${ }^{4}$, Irene Cortese ${ }^{5}$, \\ Marcus T Silva ${ }^{6}$, Steve Jacobson ${ }^{5}$, Bernardo Galvão-Castro ${ }^{3,4}$, Yoshihisa Yamano ${ }^{7}$,Graham Taylor ${ }^{8}$, \\ John Martin Bland ${ }^{1,9}$
}

From 16th International Conference on Human Retroviruses: HTLV and Related Viruses Montreal, Canada. 26-30 June 2013

\section{Background}

In the absence of an internationally recognised biomarker of treatment response in HAM/TSP, the HAM/TSP clinial trial study group chose improvement of 10 meter time walk (TW) as the primary outcome measure for the HAMLET-P trial.

\begin{abstract}
Aim
To define the minimum change in TW required for an observed treatment effect to be detectable and important. To calculate the sample size required for $90 \%$ power to detect this difference.
\end{abstract}

\section{Methods}

Prospectively collected TW (seconds/10m) of HAM/TSP patients from the four countries were submitted to two biostatisticians (Japan+UK). Analysis of covariance and $\log$ transformed TW were used.

\section{Results}

Matched TW data (baseline+6 months) were available for a total of 76 patients. Mean (SD,median) TW were 23.46 $( \pm 18.9,16.32)$ at baseline, $24.85( \pm 23.89,16.38)$ at 6 months. Mean (SD,median) $\log 10 \mathrm{~m}$ TW were $2.89( \pm 0.72,2.79)$ at baseline, $2.91( \pm 0.74,2.80)$ at 6 months. The estimated SD of $\log 10 \mathrm{~m}$ TW after adjustment for the baseline measurement was 0.26 . With 30 participants/group, we have $90 \%$ power to detect a difference of \pm 0.21 . This corresponds to a ratio of 0.81 or 1.23 , so we could detect a decrease in time of $19 \%$ or an increase of $23 \%$. With power $80 \%$ we could detect a difference of $-15 \%$ or $+18 \%$.

\section{Conclusions}

Prospectively collected longitudinal data on TW is useful in measuring inter- and intra- patient variability of this clinical efficacy marker. To power HAMLET-P at $90 \%$, a minimum of 30 patients are needed in each arm, to be increased by $3-5$ patients/arm to cover for $10-15 \%$ estimated trial drop-out rate.

\begin{abstract}
Authors' details
${ }^{1}$ Centre for Immunology and Infection, Department of Biology, Hull and York Medical School, University of York, York, UK. ${ }^{2}$ School of Pharmacy, Kitasato University, Tokyo, Japan. ${ }^{3}$ Advanced Laboratory of Public Health, Gonçalo Moniz Center, Fundação Oswaldo Cruz, Salvador, Bahia, Brazil. ${ }^{4}$ Bahiana School of Medicine and Public Health (EBMSP) Salvador, Bahia, Brazil. ${ }^{5}$ Viral Immunology Section, NINDS/NIH, Bethesda, MD, USA. ${ }^{6}$ Clinical Research Laboratory on Neuroinfection Diseases Instituto de Pesquisa Clínica Evandro Chagas Fundação Oswaldo Cruz, Fiocruz, Brazil. 'Department of Rare Diseases Research, Institute of Medical Science, St. Marianna University School of Medicine, Kanagawa, Japan. ${ }^{8}$ Section of Infectious Diseases, Faculty of Medicine, Imperial College London, London, UK. ${ }^{9}$ Department of Health Sciences, University of York, York, North Yorkshire, UK.
\end{abstract}

Published: 7 January 2014

doi:10.1186/1742-4690-11-S1-P30

Cite this article as: Martin et al:. Change in timed walk as primary outcome measure of treatment response in HAMLET-P: HAM/TSP MuLticentre Efficacy trial-Prednisolone. Retrovirology 2014 11(Suppl 1): P30

\footnotetext{
* Correspondence: fabiola.martin@hyms.ac.uk

${ }^{1}$ Centre for Immunology and Infection, Department of Biology, Hull and York Medical School, University of York, York, UK

Full list of author information is available at the end of the article
} 